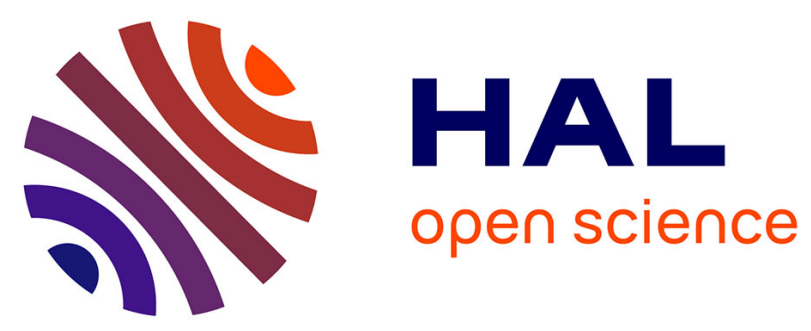

\title{
Near-field imaging of single walled carbon nanotubes emitting in the telecom wavelength range
}

F. La China, N. Caselli, F. Sarti, F. Biccari, U. Torrini, F. Intonti, A. Vinattieri, E. Durán-Valdeiglesias, C. Alonso Ramos, X. Le Roux, et al.

\section{- To cite this version:}

F. La China, N. Caselli, F. Sarti, F. Biccari, U. Torrini, et al.. Near-field imaging of single walled carbon nanotubes emitting in the telecom wavelength range. Journal of Applied Physics, 2016, 120, pp.123110. 10.1063/1.4963367 . cea-01380648

\section{HAL Id: cea-01380648 https://hal-cea.archives-ouvertes.fr/cea-01380648}

Submitted on 13 Oct 2016

HAL is a multi-disciplinary open access archive for the deposit and dissemination of scientific research documents, whether they are published or not. The documents may come from teaching and research institutions in France or abroad, or from public or private research centers.
L'archive ouverte pluridisciplinaire $\mathbf{H A L}$, est destinée au dépôt et à la diffusion de documents scientifiques de niveau recherche, publiés ou non, émanant des établissements d'enseignement et de recherche français ou étrangers, des laboratoires publics ou privés. 


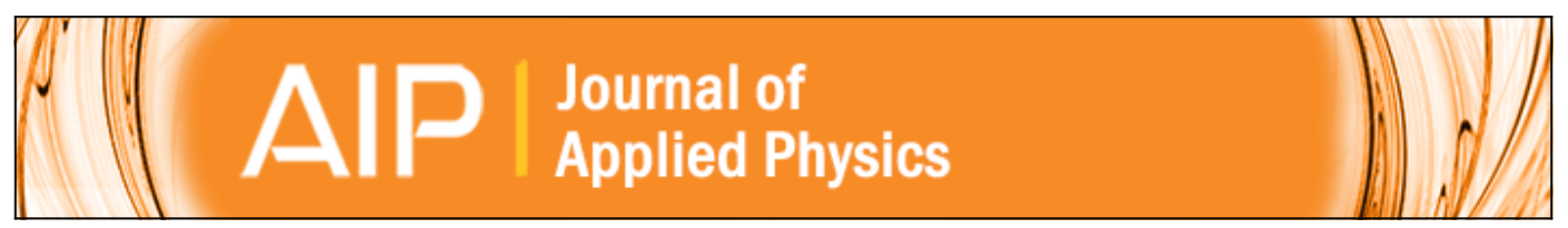

\section{Near-field imaging of single walled carbon nanotubes emitting in the telecom wavelength range}

F. La China, N. Caselli, F. Sarti, F. Biccari, U. Torrini, F. Intonti, A. Vinattieri, E. Durán-Valdeiglesias, C. Alonso

Ramos, X. Le Roux, M. Balestrieri, A. Filoramo, L. Vivien, and M. Gurioli

Citation: Journal of Applied Physics 120, 123110 (2016); doi: 10.1063/1.4963367

View online: http://dx.doi.org/10.1063/1.4963367

View Table of Contents: http://scitation.aip.org/content/aip/journal/jap/120/12?ver=pdfcov

Published by the AIP Publishing

\section{Articles you may be interested in}

Self-formation of highly aligned metallic, semiconducting and single chiral single-walled carbon nanotubes assemblies via a crystal template method

Appl. Phys. Lett. 105, 093102 (2014); 10.1063/1.4895103

On the charge transfer between single-walled carbon nanotubes and graphene

Appl. Phys. Lett. 105, 073115 (2014); 10.1063/1.4893698

Electronic transport in single-walled carbon nanotube/graphene junction

Appl. Phys. Lett. 99, 113102 (2011); 10.1063/1.3636407

Transition from single to multi-walled carbon nanotubes grown by inductively coupled plasma enhanced chemical vapor deposition

J. Appl. Phys. 110, 034301 (2011); 10.1063/1.3615945

Electron field emission from Fe-doped TiO 2 nanotubes

Appl. Phys. Lett. 96, 143102 (2010); 10.1063/1.3373607

\section{Pure Metals • Ceramics} Alloys • Polymers in dozens of forms
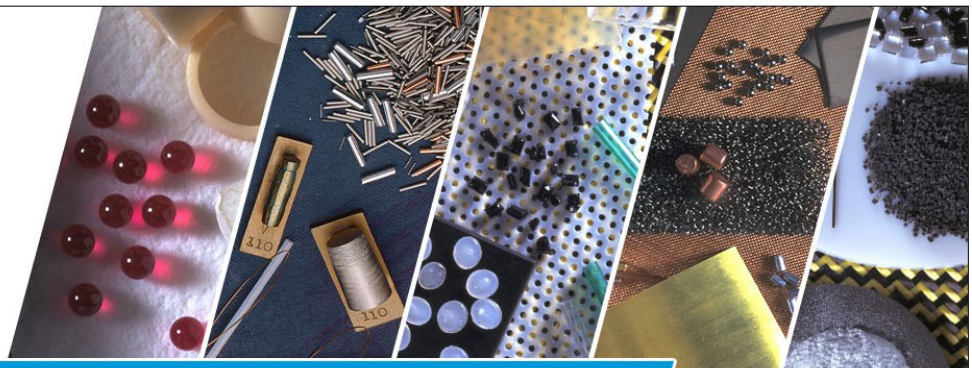

Small quantities fast Expert technical assistance $5 \%$ discount on online orders

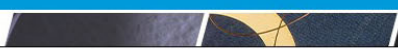




\title{
Near-field imaging of single walled carbon nanotubes emitting in the telecom wavelength range
}

\author{
F. La China, ${ }^{1,2, a)}$ N. Caselli, ${ }^{1,2}$ F. Sarti, ${ }^{1,2}$ F. Biccari, ${ }^{1,2}$ U. Torrini, ${ }^{2}$ F. Intonti, ${ }^{1,2}$ A. Vinattieri, ${ }^{1,2}$ \\ E. Durán-Valdeiglesias, ${ }^{3}$ C. Alonso Ramos, ${ }^{3}$ X. Le Roux, ${ }^{3}$ M. Balestrieri, ${ }^{4}$ A. Filoramo, ${ }^{4}$ \\ L. Vivien, ${ }^{3}$ and M. Gurioli ${ }^{1,2}$ \\ ${ }^{1}$ European Laboratory for Non-linear Spectroscopy, University of Florence, 50019 Sesto Fiorentino (FI), Italy \\ ${ }^{2}$ Department of Physics and Astronomy, University of Florence, 50019 Sesto Fiorentino (FI), Italy \\ ${ }^{3}$ Inst. Elect. Fondamentale (IEF), Univ Paris Sud, CNRS UMR 8622, Université Paris-Saclay, \\ F-91405 Orsay, France \\ ${ }^{4}$ LICSEN, NIMBE, CEA, CNRS, Université Paris-Saclay, CEA Saclay, 91191 Gif-sur-Yvette Cedex, France
}

(Received 4 May 2016; accepted 13 September 2016; published online 27 September 2016)

\begin{abstract}
Hybrid systems based on carbon nanotubes emitting in the telecom wavelength range and Si-photonic platforms are promising candidates for developing integrated photonic circuits. Here, we consider semiconducting single walled carbon nanotubes (s-SWNTs) emitting around $1300 \mathrm{~nm}$ or $1550 \mathrm{~nm}$ wavelength. The nanotubes are deposited on quartz substrate for mapping their photoluminescence in hyperspectral near-field microscopy. This method allows for a sub-wavelength resolution in detecting the spatial distribution of the emission of single s-SWNTs at room temperature. Optical signature delocalized over several micrometers is observed, thus denoting the high quality of the produced carbon nanotubes on a wide range of tube diameters. Noteworthy, the presence of both nanotube bundles and distinct s-SWNT chiralities is uncovered. Published by AIP Publishing.

[http://dx.doi.org/10.1063/1.4963367]
\end{abstract}

\section{INTRODUCTION}

Single walled carbon nanotubes (SWNTs) are cylindrical nanostructures that can be schematized as rolled single sheets of graphene owing to different angles and curvatures, resulting in different nanotube chiralities. ${ }^{1}$ Their diameter typically varies in the range of $0.4 \mathrm{~nm}-2.0 \mathrm{~nm}$, while the length can reach several micrometers. The electronic structure of SWNTs is strongly dependent on the chirality, which is related to the nanotube diameter. ${ }^{2}$ According to the chirality, both semiconducting and metallic nanotubes can be obtained, and their density of states differs from conventional 3D materials by the presence of sharp peaks (Van Hove singularities). ${ }^{1,3,4}$ Semiconducting SWNTs (s-SWNTs) are expected to play a major role in future optoelectronic devices. ${ }^{5,6}$ Due to their optical properties, carbon nanotubes represent the ideal candidate to develop on-chip active optical devices including light sources, modulators, and detectors. ${ }^{7-9}$ One of the most promising applications is the use of s-SWNTs to fabricate hybrid photonic circuits on $\mathrm{Si}$, where s-SWNTs play the role of the active optoelectronic medium in the telecom wavelength windows. ${ }^{5,10,11}$

Optical excitation of s-SWNTs generates excitons, whose recombination can be investigated at the single nanotube level using near-field photoluminescence (PL) microscopy. This technique has been used to achieve subwavelength spatial resolution and to get optical information at the nanoscale, such as the exciton diffusional range. ${ }^{12}$ Inter-nanotube transfer has been addressed in Ref. 13, and it has been suggested that efficient coupling results from carrier migration, requiring few nanometers of nanotube-nanotube

a)E-mail: lachina@1ens.unifi.it distance or even direct physical contact. Interaction with segments of DNA has been determined using near-field spectroscopy by observing the induced red shift of the exciton recombination. ${ }^{14}$ Exciton localization due to local energy landscape has also been reported. ${ }^{15}$ However, less is known of the effect of bundles on near-field emission, even if the standard picture suggests that they might quench the PL (if metallic nanotubes are present) and/or broaden the optical resonances. ${ }^{15-18}$ Note that most of the near-field experiments reported to date have been carried out for small SWNTs with emission wavelengths below the telecom windows.

Here, two different approaches are used to extract sSWNTs having their fundamental optical transition around either $1300 \mathrm{~nm}$ or $1550 \mathrm{~nm}$ wavelength, respectively. Both methods are optimized to enhance the s-SWNTs density and emission efficiency. The nanotubes are drop-casted on quartz substrates and their emission properties are studied by mapping the PL in hyperspectral near-field microscopy experiments. Using this technique, we scan the sample surface with a step size of $100 \mathrm{~nm} \times 100 \mathrm{~nm}$ while detecting $\mathrm{s}-\mathrm{SWNTs}$ emission in the $1.0 \mu \mathrm{m}-1.6 \mu \mathrm{m}$ wavelength range. The experiments, combined with far-field PL measurements, provide insights into the SWNTs spatial and spectral emission. In fact, the good optical efficiency of the selected ensemble and the sensitivity of the setup allow for detecting single s-SWNT emission even at room temperature. Effects of the interaction with the substrate and/or of strain on the sSWNTs are observed by locally evaluating the PL spectral shift. Moreover, optical emission delocalized for several $\mu \mathrm{m}$ along SWNTs is detected, thus denoting the high quality of the produced s-SWNTs. Finally, the limits of the implemented purification protocol are evaluated: the presence of 
spurious SWNT bundles is addressed by observing broad emission bands with respect to narrow emission spectra from isolated s-SWNTs.

\section{SAMPLE PREPARATION AND EXPERIMENTAL TECHNIQUES}

We analyze two kinds of SWNTs with dominant emission centered in the $1300 \mathrm{~nm}$ and $1500 \mathrm{~nm}$ telecom wavelength regions, respectively. In both cases, the pristine powder contains several carbon-based materials including metallic nanotubes. Therefore, in order to consider uniquely the optically active SWNTs (i.e., s-SWNTs), it is highly preferable to use an effective selection protocol. For the emission at $1300 \mathrm{~nm}$, we start from a commercial dry powder of HiPco carbon nanotubes. Then, we employ a purification protocol based on ultra-centrifugation of a toluene (by Sigma-Aldrich) solution containing the carbon nanotubes and the polymer Poly-9,9-di-n-octyl-fluorenyl-2,7-diyl (PFO). This process gives a high purity solution of SWNTs with $(8,6)$ and $(8,7)$ chiralities, which are known to provide room-temperature light emission in the telecom $O$ Band $(1260 \mathrm{~nm}<\lambda$ $<1360 \mathrm{~nm}$ ). ${ }^{19}$ For the emission at $1550 \mathrm{~nm}$, SWNTs (with diameter in the $1.1 \mathrm{~nm}-1.3 \mathrm{~nm}$ range) are synthesized by laser ablation (LA). ${ }^{20}$ We disperse them at the concentration of $0.2 \mathrm{mg} / \mathrm{ml}$ by sonication with poly[(9,9-dihexylfluorenyl-2,7diyl)-co-(9,10-anthracene)] (PFH-A) in toluene. The PFH-A concentration is fixed at the optimal value of $0.5 \mathrm{mg} / \mathrm{ml}$; in order to maintain these SWNTs longer for transport application, here the sonication process is quite shorter with respect to HiPco protocol. The role of polyfluorene polymers on the selective dispersion of SWNTs has been recently elucidated in Ref. 21 and the details of the purification protocols can be found in Ref. 22.

A commercial scanning near-field optical microscope (Twinsnom, by OMICRON) is used in illuminationcollection geometry for near-field PL detection. In our experimental setup, the excitation is provided by a CW Ti:Sapphire laser coupled into the near-field probe, that performs a raster scan of the sample surface at constant height, below $10 \mathrm{~nm}$. The employed near-field probe is a pure dielectric tip, made of a glass optical fiber tapered by chemical etching. ${ }^{23}$ The probe collects the SWNTs emission at each scan position, allowing for an hyperspectral mapping of the light emitters. The PL signal is dispersed by a spectrometer and collected by a nitrogen cooled InGaAs array detector. The overall spectral resolution is $0.5 \mathrm{~nm}$ and the spatial resolution, obtained from the experimental data, is of the order of $250 \mathrm{~nm}$, which allows resolving well-separated SWNTs. In order to characterize the precursor SWNT solutions, we use standard far-field PL technique and PL-excitation spectroscopy. Finally, for mapping long SWNT wires, we use a confocal microscope equipped with a Mitutoyo $100 \times$ objective $(\mathrm{NA}=0.7)$.

We deposit SWNTs onto a solid quartz substrate, using a drop cast method. The deposition is followed by thermal annealing $\left(\mathrm{T}=180^{\circ} \mathrm{C}\right)$ to produce a liquid-glass transition of the polymer and to reinforce the encapsulation of the SWNTs. Such encapsulation is meant to reduce the undesired interaction of the SWNTs with the environment that could possibly cause emission bleaching or blinking.

\section{EXPERIMENTAL RESULTS AND DISCUSSION}

The far-field PL spectrum of the HiPco SWNTs dispersed in toluene is reported in Fig. 1(a), where at least five different chiralities are observed, covering the telecom emission range from $1000 \mathrm{~nm}$ to $1400 \mathrm{~nm}$. The photoluminescence excitation (PLE) map is reported in Fig. 1(b) and allows assigning the s-SWNTs chiralities to each emission band. In particular, the HiPco SWNTs emission is dominated by the $(8,6)$ and $(8,7)$ chiralities, matching the $1300 \mathrm{~nm}$ telecom window. The spectral width turns out to be $23 \mathrm{~nm}$ for the $(8,6)$ line. A typical hyperspectral image (map dimension $5.6 \mu \mathrm{m} \times 30 \mu \mathrm{m}$ ) of a deposited HiPco solution is reported in Fig. 1(c). We observe localized bright spots characterized by different spectral features depending on the relative content of chiralities.

The emission maps of the five chiralities observed in the HiPco sample are reported in a color code that highlights the contribution for each chirality. In particular, every PL map is spectrally integrated over a given peak reported in Fig. 1(a), therefore each map corresponds to a single chirality emission band. The black areas correspond to regions where the PL signal is below the experimental noise level, therefore are interpreted as regions without optically active s-SWNTs. By

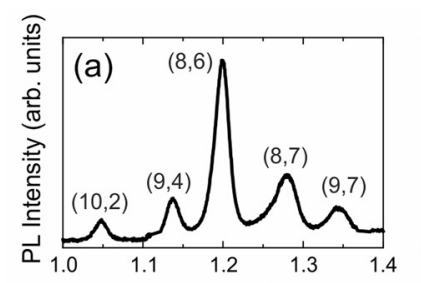

(c)
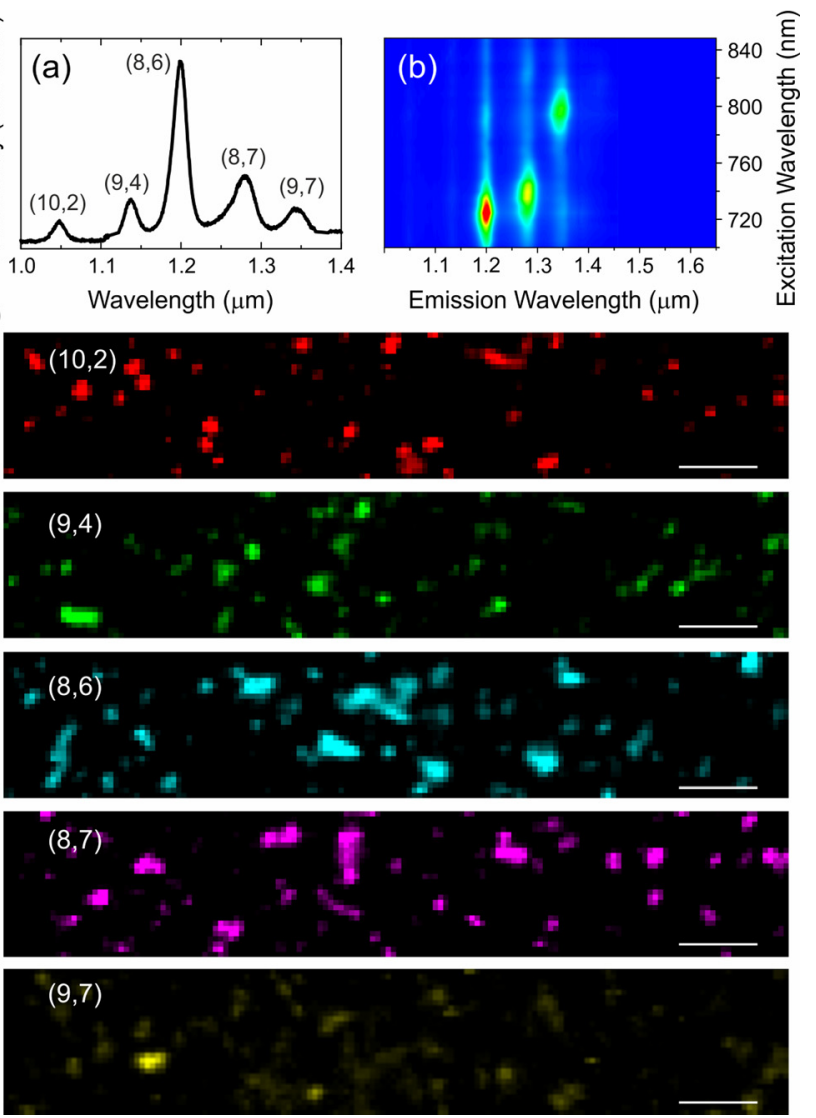

FIG. 1. HipCo s-SWNTs. (a) PL spectrum of the s-SWNTs dispersed in toluene solution. The excitation at $720 \mathrm{~nm}$ is provided by Ti: Sapphire laser. (b) photoluminescence excitation (PLE) map. (c) Near-field maps of the PL emission. Each map is spectrally integrated over a different chirality band. The white scale bar corresponds to $3 \mu \mathrm{m}$. The maps cover an area of $5.6 \mu \mathrm{m} \times 30 \mu \mathrm{m}$. 
comparing the five maps of Fig. 1(c), we find regions where different s-SWNTs overlap and regions where the PL signal originates from a single chirality. From the smallest transversal section of the bright spots, we estimate an experimental spatial resolution of the order of $250 \mathrm{~nm}$, which well agrees with the expected section of the employed dielectric tip. It is worth noting that the spatial resolution is of the order of $\lambda / 5$ at the emission wavelength of $1300 \mathrm{~nm}$.

Typical near-field PL spectra are reported in Fig. 2. The top spectrum in Fig. 2(a) corresponds to a spot where different chiralities are found, and it can be separated into independent contributions of four chiralities, each with a lineshape almost identical to the one observed in the regions where only a single chirality is present (see the lower panels). Note that the lineshape is Lorentzian denoting a homogeneous broadening; this indicates that each s-SWNT is well isolated by the polymer and then that the mutual interaction between s-SWNTs is negligible. In Fig. 2(b), we report three PL spectra of the emission band for the $(8,6)$ chirality acquired in different points. By comparing the emission bands of the dried s-SWNTs with the same bands observed in the solution (dashed line in Fig. 2(b)), we note an average wavelength red shift ( of $\approx 10 \mathrm{~nm}$ ). This shift can be attributed either to variation of the local dielectric environment (due to a re-organization of the polymer after the removal of the solvent) or to the strain induced onto the s-SWNTs by the drying process. Therefore, by analyzing the spectral response, we develop a sub-wavelength sensitive probe for investigating the local environment around the s-SWNTs.

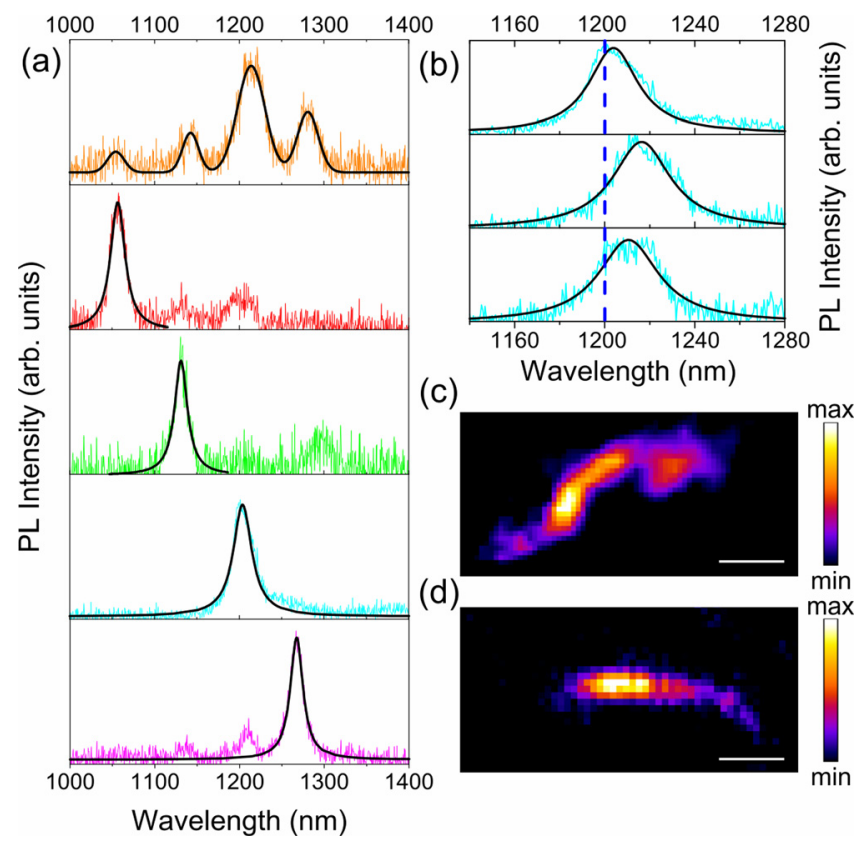

FIG. 2. (a) Typical near-field PL spectra of HiPco s-SWNTs deposited on quartz. The top spectrum corresponds to a point where all the four chiralities are found. The subsequent spectra correspond to points where a single chirality dominates. They are reported with the same color code used in Fig. 1. (b) Zoom on the $(8,6)$ emission band of three different points on the sample, denoting a spectral shift of the lineshape. The dashed line corresponds to the peak wavelength of the $(8,6)$ chirality as reported in Fig. 1. (c) and (d) High resolution PL maps of two individual HiPco s-SWNTs with specific chirality. (c) $(8,7)$ chirality; (d) $(8,6)$ chirality. The white scale bar is $1 \mu \mathrm{m}$.
In case of bright and isolated spots attributed to an individual chirality, we focus on the intensity maps of specific sSWNTs. In Figs. 2(c) and 2(d), the PL intensity distributions of two single chiralities are reported. They clearly show an elongated emission along the s-SWNT. In particular, the transverse cross-section size is of the order of the spatial resolution $(250 \mathrm{~nm})$, while the longitudinal length extends over more than two micrometers in both cases. The PL map is a measurement of the spatial distribution of the exciton emission along the nanotube length and its large longitudinal extension is a further indication of the high quality of the investigated semiconducting SWNTs. Indeed, the exciton along the nanotube is not confined in an extrinsic center, but rather delocalized over many Bohr radii. Finally, it is worth noting that the emission is quite bright along the whole length and eventually the SWNTs are slightly bent. Even though we did not perform antibunching measurements to demonstrate the quantum nature of the light emitter, we believe that our data are a sound indication that we are collecting emission from individual s-SWNTs. It is also worth stressing out that the observed room temperature emission is only $25 \mathrm{~nm}$ broad. This value has to be compared with results from other types of single emitters at room temperature such as organic molecules $(80 \mathrm{~nm}$ for dibenzoterrylene (DBT) molecules) or nitrogen-vacancy (NV) centers in diamond $(120 \mathrm{~nm}) .^{24,25}$ Such a narrow emission in the telecom spectral window is very promising for integrating SWNTs in Si-based photonic devices. ${ }^{5,6}$

Similar near-field experiments have been carried out also on LA SWNTs. The far-field PL spectrum of the LA SWNTs dispersed in toluene is reported in Fig. 3(a), where five different chiralities are observed, covering the telecom emission range from $1200 \mathrm{~nm}$ to $1600 \mathrm{~nm}$. The spectral cut observed for wavelength larger than $1600 \mathrm{~nm}$ is due to a decreased detection efficiency of the InGaAs detector. The PLE map is reported in Fig. 3(b). We find that the LA SWNTs emission is dominated by the $(12,5)$ chirality emitting at $1500 \mathrm{~nm}$ wavelength, and that the spectral width turns out to be $33 \mathrm{~nm}$ for the $(12,5)$ line. It is important to note the difference between the PL spectra of the HiPco and LA SWNTs. While the HiPco spectrum in Fig. 1(a) consists of a comb of well-distanced emission lines, the LA spectrum in Fig. 3(a) appears to contain a broadband emission superimposed with the emission of the single chiralities. We will show that this broadband contribution arises from radiative recombination in nanotube bundles, as expected due to the shorter sonication time in the LA purification.

Sub-wavelength imaging of the different chirality emission bands are reported in Fig. 3(c) (the maps cover an area of $6 \mu \mathrm{m} \times 30 \mu \mathrm{m}$ size) with different color codes for the five chiralities. High intensity spots show either emission bands corresponding to a single chirality or to overlapping chiralities or to nanotube bundles.

Typical PL spectra of LA SWNTs are reported in Fig. 4. The top spectrum in Fig. 4(a) shows the emission bands detected on a spot where four s-SWNT chiralities are found. In the other panels, we report the PL spectra of different spots where just a single chirality is observed. Similar to the data in Fig. 2(a), the emission reported in the top PL 


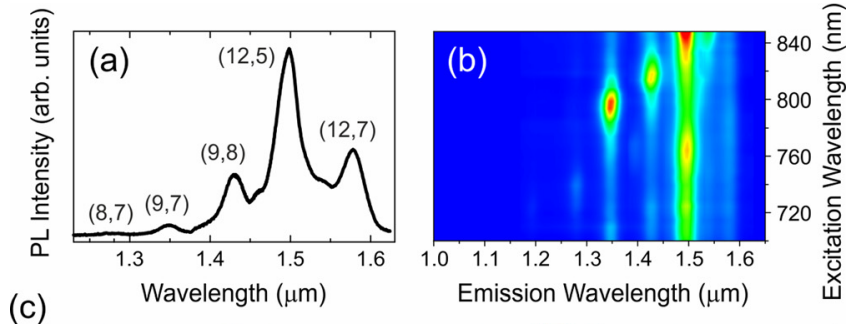

(c)
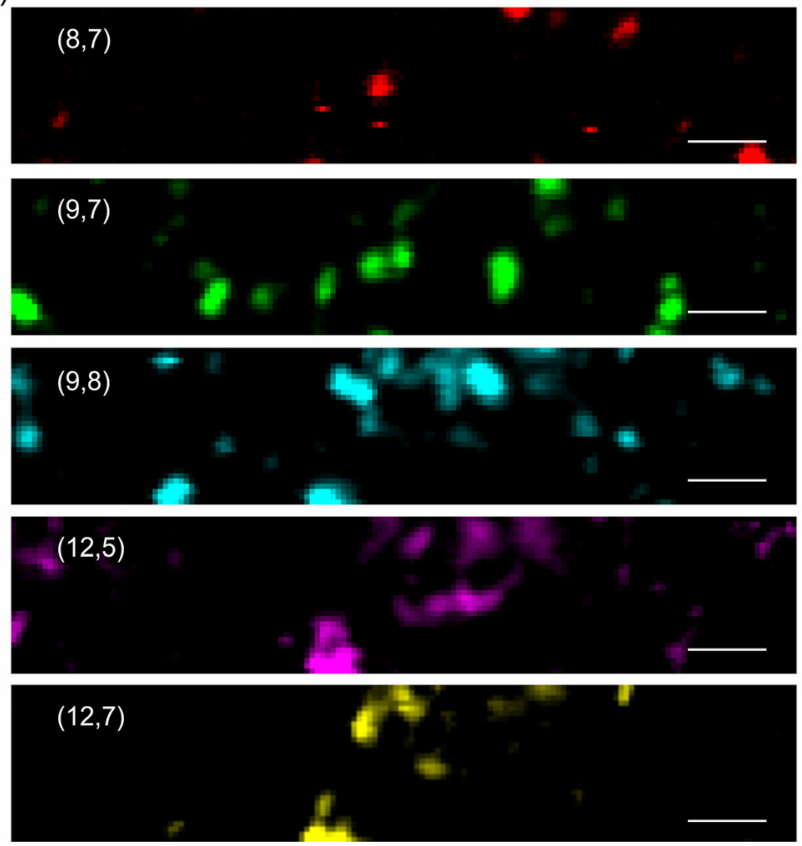

FIG. 3. LA s-SWNTs. (a) PL spectrum of the SWNTs dispersed in toluene solution. The excitation at $820 \mathrm{~nm}$ is provided by Ti: Sapphire laser. (b) PLE map. (c) Near-field maps of the PL emission integrated over different chirality bands. The white scale bar corresponds to $3 \mu \mathrm{m}$. The maps are $6 \mu \mathrm{m} \times 30 \mu \mathrm{m}$.

spectrum of Fig. 4(a) can be separated into independent contributions from individual chiralities, meaning that the sSWNTs are well isolated by the polymer. In these cases, the lineshape is Lorentzian, thus indicating the presence of well protected SWNTs. However, for LA SWNTs intensity maps, we find large bright spots also in positions where unexpected broad and non-Lorentzian spectra are observed. Several examples are given in Fig. 4(b). The peaks of these bands are not straightforwardly identified as a simple overlap between defined chiralities. Since the PL is a measure of the joint density of states of the conduction and valence bands, we conclude that strong modifications of the electronic structures are needed in order to produce the PL spectra reported in Fig. 4(b).

The simplest explanation could be that we are mapping large nanotube bundles comprising nanotubes of different chiralities that are (locally) in direct contact with one another. Since the electronic states overlap and hybridize in the region where the contact occurs, excitons can move (unidirectionally) from nanotubes with a higher gap to other with smaller gap and then recombine at a lower energy. Therefore, the near-field optical spectroscopy can discriminate between bundles of SWNTs, where the electronic states interact producing broad PL bands, and agglomerate of s-SWNTs in which each nanotube is isolated by the encapsulating polymer and gives rise to a comb of individual PL bands.

In order to corroborate the idea that nanotube bundles are responsible for the broad PL band, we exploit the accidental production of macroscopic wires composed of SWNTs and polymer. As schematically reported in Fig. 5(a), this type of wire is formed by slowly extracting a substrate from a water bath on top of which a small amount (few tens of $\mu \mathrm{l}$ ) of toluene solution containing PFH-A and highly concentrated nanotubes is drying. The deposited film onto the substrate surface (typically $\mathrm{SiO}_{2}$ ) bridges to the liquid surface with a macroscopic wire composed of polymer and SWNT. The thickness of the nanotube wire is of the order of $50 \mu \mathrm{m}$ (SEM image is reported in Fig. 5(b)) and the total length is several $\mathrm{mm}$. The SWNT wires have some conceptual and morphological similarities with those reported in the literature. ${ }^{26}$ During the elongation process, it is very likely
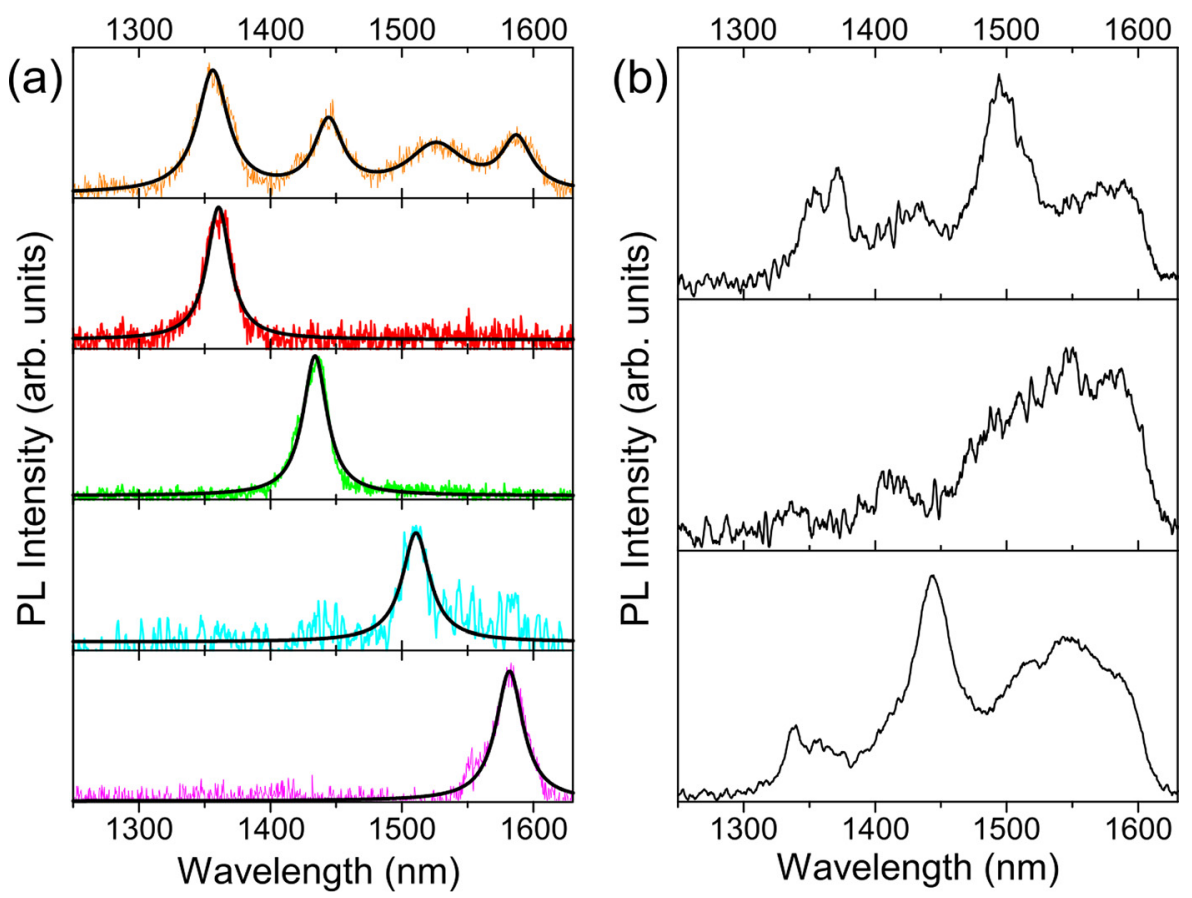

FIG. 4. (a) Typical near-field PL spectra of LA SWNTs. The different chiralities are reported with different colors, which correspond to those of Fig. 3. (b) Details of the broad emission bands are found in bright PL intensity spots. 
(a)

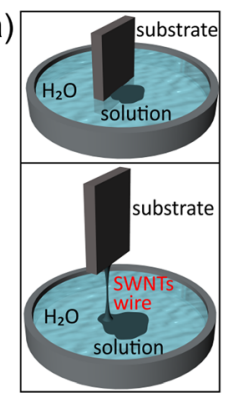

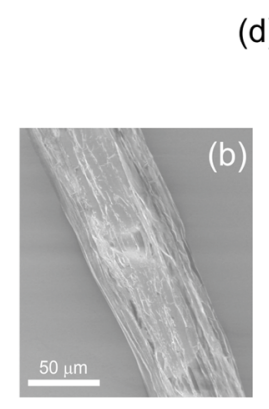

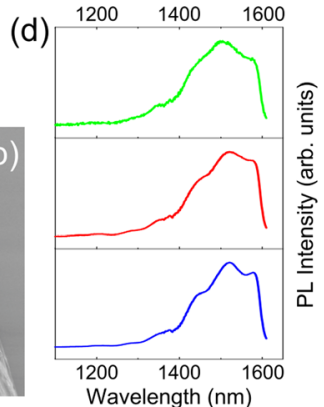

(c)

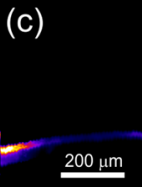

FIG. 5. (a) Scheme of the wire formation: an immersed substrate is slowly lifted up. (b) SEM image of the SWNT wire. (c) Micro-PL map of the macroscopic SWNT wire, spectrally integrated from $1200 \mathrm{~nm}$ to $1600 \mathrm{~nm}$. The wire, after deposition on a quartz substrate, is slightly bended. (d) Spectra collected on different spots along the wire in (c).

that the polymer is partially swiped away favoring the formation of large and long bundles of aligned and interacting nanotubes. The intensity map of one of these wires (acquired with the far-field micro-PL setup) integrated over the whole PL spectrum is reported in Fig. 5(c). From these data, we observe that bright spots are macroscopically elongated along the wire. The PL lineshape is very similar in all the points of the macroscopic wire, as highlighted in Fig. 5(d). These spectra show a very broad, non-Lorentzian and weakly modulated PL band in which single chiralities cannot be resolved. Therefore, we conclude that bundles are characterized by SWNT hybridization leading to broad band PL emission, in agreement with the hypothesis made when discussing the near-field data.

\section{CONCLUSIONS}

In conclusion, we demonstrated that infrared hyperspectral near-field imaging allows for a deep investigation of the morphology and optical properties of SWNTs in broad band telecom spectral regions. By mapping the photoluminescence with sub-wavelength spatial resolution, the distribution of each individual chirality is observed, as well as the effect induced by the strain on each s-SWNT. In addition, we retrieve the presence of nanotube bundles, which results in broad emission bands due to electronic states hybridization. Our results highlight the importance of sub-wavelength spectroscopy to assess the properties of SWNTs. Noteworthy, the observed narrow emission in the telecom window is promising for integrating s-SWNTs in Si-based photonic devices.

\section{ACKNOWLEDGMENTS}

This work was supported by the FET Project No. FP7 618025 CARTOON. We acknowledge the support by Marco De Pas for developing and fixing the electronics of the SNOM setup.
${ }^{1}$ R. Saito, G. Dresselhaus, and M. S. Dresselhaus, Physical Properties of Carbon Nanotubes (Imperial College Press, 1998).

${ }^{2}$ R. Saito, M. Fujita, G. Dresselhaus, and M. S. Dresselhaus, "Electronic structure of chiral graphene tubules," Appl. Phys. Lett. 60, 2204-2206 (1992).

${ }^{3}$ P. Kim, T. W. Odom, J.-L. Huang, and C. M. Lieber, "Electronic density of states of atomically resolved single-walled carbon nanotubes: Van Hove singularities and end states," Phys. Rev. Lett. 82, 1225 (1999).

${ }^{4}$ J. W. G. Wilder, L. C. Venema, A. G. Rinzler, R. E. Smalley, and C. Dekker, "Electronic structure of atomically resolved carbon nanotubes," Nature 391, 59-62 (1998).

${ }^{5}$ A. Noury, X. Le Roux, L. Vivien, and N. Izard, "Controlling carbon nanotube photoluminescence using silicon microring resonators," Nanotechnology 25(21), 215201 (2014).

${ }^{6}$ E. Gaufrès, N. Izard, A. Noury, X. L. Roux, G. Rasigade, A. Beck, and L. Vivien, "Light emission in silicon from carbon nanotubes," ACS Nano 6(5), 3813-3819 (2012).

${ }^{7}$ A. Högele, C. Galland, M. Winger, and A. Imamoglu, "Band gap fluorescence from individual single-walled carbon nanotubes," Phys. Rev. Lett. 100, 217401 (2008).

${ }^{8}$ R. H. Baughman, A. Zakhidov, and W. A. de Heer, "Carbon nanotubes the route toward applications," Science 297, 787 (2002).

${ }^{9}$ P. Avouris, M. Freitag, and V. Perebeinos, "Carbon-nanotube photonics and optoelectronics," Nat. Photonics 2, 341-350 (2008).

${ }^{10}$ S. Fan, W. Liang, H. Dang, N. Franklin, T. Tombler, M. Chapline, and H. Dai, "Carbon nanotube arrays on silicon substrates and their possible application," Physica E 8(2), 179-183 (2000).

${ }^{11}$ F. La China, F. Intonti, N. Caselli, F. Lotti, F. Sarti, A. Vinattieri, A. Noury, X. Le Roux, W. Zhang, E. Cassan, C. Alonso Ramos, E. Durán Valdeiglesias, N. Izard, L. Vivien, and M. Gurioli, "Near-field fanoimaging of TE and TM modes in silicon microrings," ACS Photonics 2(12), 1712-1718 (2015).

${ }^{12}$ L. Cognet, A. D. Tsyboulski, J.-D. R. Rocha, C. Doyle, J. Tour, and R. B. Weisman, "Stepwise quenching of exciton fluorescence in carbon nanotubes by single-molecule reactions," Science 316(5830), 1465-1468 (2007).

${ }^{13}$ H. Qian, C. Georgi, N. Anderson, A. A. Green, M. C. Hersam, L. Novotny, and A. Hartschuh, "Exciton energy transfer in pairs of singlewalled carbon nanotubes," Nano Lett. 8(5), 1363-1367 (2008).

${ }^{14}$ H. Qian, P. T. Araujo, C. Georgi, T. Gokus, N. Hartmann, A. A. Green, A. Jorio, M. C. Hersam, L. Novotny, and A. Hartschuh, "Visualizing the local optical response of semiconducting carbon nanotubes to DNA-wrapping," Nano Lett. 8(9), 2706-2711 (2008).

${ }^{15}$ C. Georgi, A. A. Green, M. C. Hersam, and A. Hartschuh, "Probing exciton localization in single-walled carbon nanotubes using high-resolution near-field microscopy," ACS Nano 4(10), 5914-5920 (2010).

${ }^{16}$ M. J. O’Connell, S. J. Bachilo, C. B. Huffman, V. C. Moore, M. S. Strano, E. H. Haroz, K. L. Rialon, P. J. Boul, W. H. Noon, C. Kittrell, J. Ma, R. H. Hauge, B. Weisman, and R. H. Smal, "Band gap fluorescence from individual single-walled carbon nanotubes," Science 297(5581), 593-596 (2002).

${ }^{17}$ A. Hartschuh, H. N. Pedrosa, L. Novotny, and T. D. Krauss, "Simultaneous fluorescence and Raman scattering from single carbon nanotubes," Science 301(5638), 1354 (2003).

${ }^{18}$ J. Lefebvre, S. Maruyama, and P. Finnie, "Photoluminescence: Science and application," in Carbon Nanotubes: Advanced Topics in the Synthesis, Structure, Properties and Applications, Springer Topics in Applied Physics (Springer Berlin, Heidelberg, 2008), pp. 287-319.

${ }^{19}$ A. Nish, J. Hwang, J. Doig, and R. J. Nicholas, "Highly selective dispersion of single-walled carbon nanotubes using aromatic polymers," Nat. Nanotechnol. 2(10), 640-646 (2007).

${ }^{20}$ A. Gorbunov, R. Friedlein, O. Jost, M. Golden, J. Fink, and W. Pompe, "Gas-dynamic consideration of the laser evaporation synthesis of singlewall carbon nanotubes,” Appl. Phys. A 69(1), S593-S596 (1999).

${ }^{21}$ H. Yang, V. Bezugly, J. Kunstmann, A. Filoramo, and G. Cuniberti, "Diameter-selective dispersion of carbon nanotubes via polymers: A competition between adsorption and bundling," ACS Nano 9, 9012 (2015).

${ }^{22}$ N. Izard, S. Kazaoui, K. Hata, T. Okazaki, T. Saito, S. Iijima, and N. Minami, "Semiconductor-enriched single wall carbon nanotube networks applied to field effect transistors," Appl. Phys. Lett. 92(24), 243112 (2008). 
${ }^{23}$ R. Stöckle, C. Fokas, V. Deckert, R. Zenobi, B. Sick, B. Hecht, and U. Wild, "High-quality near-field optical probes by tube etching," Appl. Phys. Lett. 75(2), 160-162 (1999).

${ }^{24}$ A. Gruber, A. Dräbenstedt, C. Tietz, L. Fleury, J. Wrachtrup, and C. von Borczyskowski, "Scanning confocal optical microscopy and magnetic resonance on single defect centers," Science 276, 2012 (1997).
${ }^{25}$ C. Toninelli, K. Early, J. Bremi, A. Renn, S. Götzinger, and V. Sandoghdar, "Near-infrared single-photons from aligned molecules in ultrathin crystalline films at room temperature," Opt. Express 18, 6577-6582 (2010).

${ }^{26} \mathrm{P}$. Poulin, B. Vigolo, and P. Launois, "Films and fibers of oriented single wall nanotubes," Carbon 40(10), 1741-1749 (2002). 